\title{
Intestinal Volvulus
}

National Cancer Institute

\section{Source}

National Cancer Institute. Intestinal Volvulus. NCI Thesaurus. Code C98963.

Twisting of a loop of bowel that results in intestinal obstruction. 